\title{
THE ASYMPTOTIC FORMULAE IN THE PROBLEM ON CONSTRUCTING HYPERBOLICITY AND STABILITY REGIONS OF DYNAMICAL SYSTEMS
}

\author{
L.S. IBRAGIMOVA, I.ZH. MUSTAFINA, M.G. YUMAGULOV
}

\begin{abstract}
The paper proposes a new general method allowing us to study the problem on constructing hyperbolicity and stability regions for nonlinear dynamical systems. The method is based on a modification of the method by M. Rozo for studying the stability of linear systems with periodic coefficients depending on a small parameter and on the asymptotic formulae in the perturbation theory of linear operators. We obtain approximate formulae describing the boundary of hyperbolicity and stability regions. As an example, we provide the scheme for constructing the stability regions for Mathieu equation.
\end{abstract}

Keywords: hyperbolicity regions, stability regions, dynamical systems, small parameter, asymptotic formula

Mathematics Subject Classification: 34D20, 37C60

\section{INTRODUCTION}

We consider the equation

$$
\frac{d x}{d t}=A(\mu, t) x+a(x, t, \mu), \quad x \in \mathbb{R}^{N}, \mu \in \mathbb{R}^{k},
$$

where the matrix $A(\mu, t)$ and the vector-function $a(x, t, \mu)$ are continuous and $T$-periodic in $t$ being smooth (continuously differentiable) w.r.t. scalar or vector parameter $\mu$. At that, $a(x, t, \mu)$ depends smoothly on $x$ and satisfies the condition

$$
\|a(x, t, \mu)\|=O\left(\|x\|^{2}\right) \text { as }\|x\| \rightarrow 0
$$

uniformly in $t$ and $\mu$. Hereinafter the symbol $\|\cdot\|$ stands for the Euclidean norms of vectors and matrices.

For all values of the parameter $\mu$, equation (1) has the equilibrium point $x=0$. We denote by $p_{-}, p_{0}$ and $p_{+}$the number of the multiplicators (counting multiplicities) of the linear $T$-periodic system

$$
\frac{d x}{d t}=A(\mu, t) x, \quad x \in \mathbb{R}^{N},
$$

whose absolute value is less than, equal to or greater than 1 , respectively; then $p_{-}+p_{0}+p_{+}=N$. The triple $\left(p_{-}, p_{0}, p_{+}\right)$is called [1]-[4] a topological type of the equilibrium point $x=0$ of system (1). We also say that the equilibrium point $x=0$ is hyperbolic if $p_{0}=0$; otherwise it is called non-hyperbolic.

An open connected set $G$ in the space of the parameters $\mathbb{R}^{k}$ is called a hyperbolicity region of the equilibrium point $x=0$ of system (1) if for each $\mu \in G$ the equilibrium point $x=0$ is hyperbolic with the same topological type $\left(p_{-}, 0, p_{+}\right)$. The set $G$ is called a stability region of the

L.S. Ibragimova, I.Zh. Mustafina, M.G. Yumagulov, The Symptotical formulas for the ProbLEM OF THE CONSTRUCTION HYPERBOLIC AND STABILITY REGIONS OF DYNAMICAL SYSTEMS.

(c) Ibragimova L.S., Mustafina I.Zh., Yumagulov M.G. 2016.

Submitted March 24, 2016. 
equilibrium point $x=0$ of system (1) if for each $\mu \in G$ the equilibrium point $x=0$ is stable. In a natural way we introduce the notions of asymptotic stability region and an instability region.

In what follows by $\mathcal{B}\left(\mu_{0}, \delta_{0}\right)$ we denote an open ball of radius $\delta_{0}>0$ centered at a point $\mu_{0}$ in the space of the parameters $\mu \in \mathbb{R}^{k}$.

If the equilibrium point $x=0$ of system (1) is hyperbolic for some $\mu=\mu_{0}$ and $\left(p_{-}, 0, p_{+}\right)$is its topological type, there exists a ball $\mathcal{B}\left(\mu_{0}, \delta_{0}\right)$ such that for all $\mu \in \mathcal{B}\left(\mu_{0}, \delta_{0}\right)$ the equilibrium point $x=0$ is hyperbolic with the same topological type $\left(p_{-}, 0, p_{+}\right)$.

If as $\mu=\mu_{0}$, the equilibrium point $x=0$ is non-hyperbolic, as a rule, its topological type changes as $\mu$ passes $\mu_{0}$. Namely, as a rule, in the space $\mathbb{R}^{k}$ of parameters $\mu$, one or several manifolds $\gamma_{j}(j=1, \ldots, m)$ of codimension 1 pass the point $\mu_{0}$, and these manifolds partition some ball $\mathcal{B}\left(\mu_{0}, \delta_{0}\right)$ into $2 m$ hyperbolicity subregions $\mathcal{B}_{k}$.

In particular, if as $\mu=\mu_{0}$, the equilibrium point $x=0$ has a topological type $\left(p_{-}, p_{0}, 0\right)$, where $p_{0} \geqslant 1$, then the hyperbolicity regions $\mathcal{B}_{k}$ can be stability regions $\mathcal{B}_{j}^{s}$ and instability regions $\mathcal{B}_{j}^{n}$ (alternating in a natural sense) for the solution $x=0$ of system $(1)$.

The problem on constructing hyperbolicity regions and, in particular, the stability and instability regions of solutions to differential equations is one of the important and interesting problems in linear and nonlinear dynamics and the applications. Here effective methods of studying were proposed, a series of important from the theoretical and practical points of views problems were solved (see, for instance, 4]-[7] and the references therein). It should be noted that most of known works concern autonomous equations [8]-[12]. These problems are much less studied for non-autonomous equations with periodic coefficients, although many important theoretical and practical issues lead one to such problems. The main point is a complicated problem of constructing multiplicators; they can be constructed explicitly only in simplest cases. Here the known results concern studying particular equations (see [6, 7], [13]-[15]).

In the present paper we propose a new general approach allowing us to obtain approximate formulae in the problem of constructing the manifolds $\gamma_{j}$ and the hyperbolicity regions $\mathcal{B}_{k}$ of system (1). The approach is based on a modification of a method by M. Roseau [16] and on asymptotic formulae in the perturbation theory of linear operators [17, 18]. The main results are obtained under the assumption that the following conditions hold:

a) as $\mu=\mu_{0}$, the matrix $A_{0} \equiv A\left(\mu_{0}, t\right)$ is independent of $t$;

b) the matrix $A_{0}$ has a simple zero eigenvalue or a pair of simple eigenvalues $\pm i \omega_{0}, \omega_{0}>0$, while its other eigenvalues have non-zero real parts.

Then, as $\mu=\mu_{0}$, the equilibrium point $x=0$ of system (1) is non-hyperbolic and has a topological type $\left(p_{-}, p_{0}, p_{+}\right)$, where $p_{-}, p_{0}$ and $p_{+}$is the number of the eigenvalues of the matrix $A_{0}$ with negative, zero and positive real parts, respectively. At that, $p_{0}=1$ or $p_{0}=2$.

The approach proposed in the paper can be modified for the stated problems under conditions more general than a) and b). For example, for the situations, when the matrix $A_{0}$ has several pairs of pure imaginary eigenvalues or when the matrix $A_{0}$ has pure imaginary degenerate eigenvalues and so forth.

Under an appropriate modification, we can obtain the analogues of the below results for the equations of form (1) defined in the complex plane $\mathbb{C}^{N}$ with complex matrix $A(\mu, t)$, complex vector-function $a(x, t, \mu)$ and complex parameter $\mu$.

\section{MAIN RESUlTS}

Condition b) can be realized in one of the following cases:

$1^{0}$. $A_{0}$ has a simple zero eigenvalue;

$2^{0}$. $A_{0}$ has a pair of simple eigenvalues $\pm i \omega_{0}$, where $\omega_{0}>0$ and $\omega_{0} \neq \frac{\pi k}{T}, k$ is a natural number;

$3^{0}$. $A_{0}$ has a pair of simple eigenvalues $\pm i \omega_{0}$, where $\omega_{0}=\frac{\pi k_{0}}{T}$ for some natural $k_{0}$. 
Here in all three cases we assume that other eigenvalues of the matrix $A_{0}$ have non-zero real parts.

From the point of view of the general bifurcation theory (see, for instance, [1, 2, 19]), in all aforementioned cases, the equilibrium point $x=0$ of equation (1) is non-hyperbolic, while the value $\mu_{0}$ of the parameter $\mu$ is a bifurcation point. At that, in the cases $1^{0}$ and $2^{0}$, the codimension of the bifurcation is equal to one, while in the case $3^{0}$ it is two. This is why, in the first two cases, the parameter $\mu$ is scalar, while in the third case it is two-dimensional.

Hereinafter (where it will produce no ambiguity), for the sake of brevity, we shall employ the same notation for a square matrix of order $N$ and the associated linear operator acting in the standard basis of the $N$-dimensional real space $\mathbb{R}^{N}$ or the $N$-dimensional complex space $\mathbb{C}^{N}$.

In what follows we shall make use of the following notions corresponding to the above definitions. A square matrix $A$ is called hyperbolic if it has no pure imaginary eigenvalues and a non-hyperbolic otherwise. Let a matrix $A$ has $p_{-}, p_{0}$ and $p_{+}$eigenvalues counting multiplicities with a negative, zero and positive real parts, respectively. Then the triple $\left(p_{-}, p_{0}, p_{+}\right)$is called a topological type of the matrix $A$. These notions also concern a linear operator acting in a finite-dimensional linear space.

In the below constructions we employ essentially the following auxiliary statement.

Consider the equation

$$
\frac{d x}{d t}=A(t) x+a(x, t), \quad x \in \mathbb{C}^{N},
$$

where $A(t)$ is a continuous $T$-periodic matrix and the function $a(x, t)$ is continuous in $t$, continuously differentiable in $x$ and satisfies the relations: $a(x, t+T) \equiv a(x, t),\|a(x, t)\|=O\left(\|x\|^{2}\right)$ as $\|x\| \rightarrow 0$. Let $U(t)$ be a non-degenerate continuous $T$-periodic matrix. The matrices $A(t)$ and $U(t)$, as well as the function $a(x, t)$ can be both real and complex.

The change $y=U(t) x$ reduces equation (3) to

$$
\frac{d y}{d t}=B(t) y+b(y, t), \quad y \in \mathbb{C}^{N}
$$

where

$$
B(t)=U^{\prime}(t) U^{-1}(t)+U(t) A(t) U^{-1}(t), \quad b(y, t)=U(t) a\left(U^{-1}(t) y, t\right) .
$$

Lemma 1. A non-degenerate T-periodic transformation $y=U(t) x$ keeps the topological type of the equilibrium point $x=0$ of system (3), that is, the topological types of the zero equilibrium points of systems (3) and (4) are same.

The proof of this and others statements are provided in the end of the paper.

2.1. Case $1^{0}$. Assuming that $\mu$ is a scalar parameter (that is, $\mu \in \mathbb{R}$ ) and letting for the sake of simple notations $\mu_{0}=0$, we rewrite equation (1) as

$$
\frac{d x}{d t}=\left[A_{0}+\mu A_{1}(t)+A_{2}(\mu, t)\right] x+a(x, t, \mu) .
$$

where $A_{1}(t)=A_{\mu}^{\prime}(0, t)$, and the matrix $A_{2}(\mu, t)$ satisfies the relation $\left\|A_{2}(\mu, t)\right\|=O\left(\mu^{2}\right)$ as $\mu \rightarrow 0$ uniformly in $t$. Equation (5) can be considered as an equation with a scalar parameter $\mu$ and a $T$-periodic in $t$ right hand side.

Since in the considered case the space of the parameters $\mu$ is one-dimensional, the aforementioned manifolds $\gamma_{j}$ degenerate into the point $\mu_{0}=0$. At that, the hyperbolicity regions $\mathcal{B}_{k}$ are the intervals of the form $\left(-\delta_{0}, 0\right)$ or $\left(0, \delta_{0}\right)$ for some $\delta_{0}>0$.

We denote by $e_{0}$ and $g_{0}$ the eigenvectors of the matrix $A_{0}$ and the transposed matrix $A_{0}^{*}$, respectively, associated with the eigenvalue 0 . These vectors can be normalized: $\left\|e_{0}\right\|=1$ and $\left(e_{0}, g_{0}\right)=1$. Hereinafter the symbol $(\cdot, \cdot)$ stands for the scalar product in the spaces $\mathbb{R}^{N}$ and $\mathbb{C}^{N}$. 
We let

$$
\lambda_{1}=\int_{0}^{T}\left(A_{1}(t) e_{0}, g_{0}\right) d t .
$$

Theorem 1. Let a matrix $A_{0}$ has a simple zero eigenvalue and the topological type of this matrix is $\left(p_{-}, 1, p_{+}\right)$. Let $\lambda_{1} \neq 0$. Then the topological type of the zero equilibrium of system (5) is equal to $\left(1+p_{-}, 0, p_{+}\right)$for all sufficiently small $|\mu|$ such that $\mu \lambda_{1}<0$; it is equal to $\left(p_{-}, 0,1+p_{+}\right)$if $\mu \lambda_{1}>0$.

This statement implies

Theorem 2. Let the matrix $A_{0}$ has a simple zero eigenvalue and other eigenvalues of the matrix $A_{0}$ have negative real parts. Let $\lambda_{1} \neq 0$. Then the solution $x=0$ of equation (5) is asymptotically stable for all small $|\mu|$ such that $\mu \lambda_{1}<0$; it is unstable if $\mu \lambda_{1}>0$.

Corollary 1. Let $\lambda_{1} \neq 0$. Then under the assumptions of Theorem 1, for some $\delta_{0}>0$, the intervals $\left(-\delta_{0}, 0\right)$ and $\left(0, \delta_{0}\right)$ are hyperbolicity regions of the solution $x=0$ to equation (5) (with different topological type). Let $\lambda_{1}>0\left(\lambda_{1}<0\right)$. Then under the assumptions of Theorem 2 the interval $\left(-\delta_{0}, 0\right)\left(\left(0, \delta_{0}\right)\right)$ is an asymptotic stability region, while the interval $\left.\left(0, \delta_{0}\right)\left(\left(-\delta_{0}, 0\right)\right)\right)$ is an instability region of the solution $x=0$ to equation (5).

2.2. Case $2^{0}$. As in the case $1^{0}$, here it is natural to assume that $\mu$ is a scalar parameter and for the sake of simplicity of notations $\mu_{0}=0$. In other words, here equation (1) can be also represented as (5), while the hyperbolicity region $\mathcal{B}_{k}$ are the intervals of the form $\left(-\delta_{0}, 0\right)$ or $\left(0, \delta_{0}\right)$ for some $\delta_{0}>0$.

Since the matrix $A_{0}$ has eigenvalues $\pm i \omega_{0}$, there exist non-zero vectors $e, g, e^{*}, g^{*} \in \mathbb{R}^{N}$ satisfying the identities:

$$
A_{0}(e+i g)=i \omega_{0}(e+i g), \quad A_{0}^{*}\left(e^{*}+i g^{*}\right)=-i \omega_{0}\left(e^{*}+i g^{*}\right) .
$$

These vectors can be normalized:

$$
\|e\|=\|g\|=1,\left(e, e^{*}\right)=\left(g, g^{*}\right)=1,\left(e, g^{*}\right)=\left(g, e^{*}\right)=0 .
$$

We let

$$
\chi_{1}=\int_{0}^{T}\left[\left(A_{1}(t) e, e^{*}\right)+\left(A_{1}(t) g, g^{*}\right)\right] d t .
$$

Theorem 3. Let the matrix $A_{0}$ has a pair of simple eigenvalues $\pm i \omega_{0}$, where $\omega_{0}>0$ and $\omega_{0} \neq \frac{\pi k}{T}$ ( $k$ is a natural number) and let the topological type of this matrix is equal to $\left(p_{-}, 2, p_{+}\right)$. Let $\chi_{1} \neq 0$. Then the topological type of the zero equilibrium point of system (5) is equal to $\left(2+p_{-}, 0, p_{+}\right)$for all small $|\mu|$ such that $\mu \chi_{1}<0$; it is equal to $\left(p_{-}, 0,2+p_{+}\right)$if $\mu \chi_{1}>0$.

This statement implies

Theorem 4. Let the matrix $A_{0}$ has a pair of simple eigenvalues $\pm i \omega_{0}$, where $\omega_{0}>0$ and $\omega_{0} \neq \frac{\pi k}{T}$ ( $k$ is a natural number), while other eigenvalues of the matrix $A_{0}$ have negative real parts. Let $\chi_{1} \neq 0$. Then the solution $x=0$ of system $(5)$ is asymptotic stable for all small $|\mu|$ such that $\mu \chi_{1}<0$; it is unstable if $\mu \chi_{1}>0$.

Corollary 2. Let $\chi_{1} \neq 0$. Then under the assumptions of Theorem 3 for some $\delta_{0}>0$ the intervals $\left(-\delta_{0}, 0\right)$ and $\left(0, \delta_{0}\right)$ are hyperbolicity regions of the solution $x=0$ to equation (5) (with different topological types). Let $\chi_{1}>0\left(\chi_{1}<0\right)$. Then under the assumptions of Theorem 4 the interval $\left(-\delta_{0}, 0\right)\left(\left(0, \delta_{0}\right)\right)$ is an asymptotic stability region, while the interval $\left.\left(0, \delta_{0}\right)\left(\left(-\delta_{0}, 0\right)\right)\right)$ is an instability region of the solution $x=0$ of equation (5). 


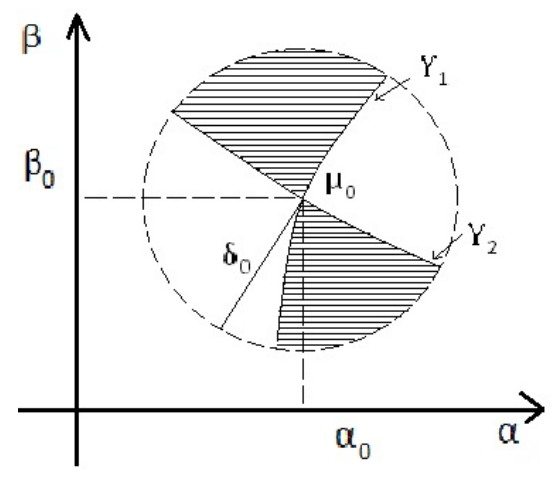

FiguRE 1. Hyperbolicity regions in the plane of the parameters $(\alpha, \beta)$

2.3. Case $3^{0}$. The case $3^{0}$ is the most complicated. Here it is natural to assume that $\mu$ is a two-dimensional parameter. Let $\mu=(\alpha, \beta)$, where $\alpha$ and $\beta$ are scalar real parameters and $\mu_{0}=\left(\alpha_{0}, \beta_{0}\right)$. Then equation (1) becomes

$$
\frac{d x}{d t}=A(\alpha, \beta, t) x+a(x, t, \alpha, \beta), \quad x \in \mathbb{R}^{N} .
$$

We assume that the entried of the matrix $A(\alpha, \beta, t)$ are twice continuously differentiable in $\alpha$ and $\beta$. Then it can be represented as

$$
\begin{aligned}
A(\alpha, \beta, t)= & A_{0}+\left(\alpha-\alpha_{0}\right) B_{1}(t)+\left(\beta-\beta_{0}\right) B_{2}(t)+\left(\alpha-\alpha_{0}\right)^{2} B_{11}(t) \\
& +\left(\beta-\beta_{0}\right)^{2} B_{22}(t)+\left(\alpha-\alpha_{0}\right)\left(\beta-\beta_{0}\right) B_{12}(t)+B_{3}(\alpha, \beta, t),
\end{aligned}
$$

where $A_{0}$ is a constant matrix (we recall the assumption that the matrix $A\left(\alpha_{0}, \beta_{0}, t\right)$ is independent of $t)$, the matrices $B_{j}(t), B_{i j}(t)$ and $B_{3}(\alpha, \beta, t)$ are $T$-periodic in $t$ and the matrix $B_{3}(\alpha, \beta, t)$ satisfies the relation

$$
\left\|B_{3}(\alpha, \beta, t)\right\|=O\left(\left(\alpha-\alpha_{0}\right)^{2}+\left(\beta-\beta_{0}\right)^{2}\right)^{3 / 2} \text { as }(\alpha, \beta) \rightarrow\left(\alpha_{0}, \beta_{0}\right),
$$

uniformly in $t$. Since in the considered case the space $\mathbb{R}^{2}$ of the parameters $\mu$ is two-dimensional, the aforementioned manifolds $\gamma_{j}$ are, as a rule, smooth curves passing the point $\mu_{0}=\left(\alpha_{0}, \beta_{0}\right)$, while the hyperbolicity regions $\mathcal{B}_{k}$ of the solution $x=0$ of equation $(10)$ are open subregions of some ball $\mathcal{B}\left(\mu_{0}, \delta_{0}\right) \subset \mathbb{R}^{2}$ and the curves $\gamma_{j}$ form the boundaries of the regions $\mathcal{B}_{k}$ (see a corresponding example in Figure 1).

In what follows, we define the boundary of a hyperbolicity region in the vicinity of the point $\left(\alpha_{0}, \beta_{0}\right)$ as a smooth curve $\Upsilon$ passing the point $\mu_{0}=\left(\alpha_{0}, \beta_{0}\right)$ such that:

- for each $(\alpha, \beta) \in \Upsilon$, the solution $x=0$ of the equation 10 is non-hyperbolic with the same topological type;

- for each point $(\alpha, \beta) \in \Upsilon,(\alpha, \beta) \neq\left(\alpha_{0}, \beta_{0}\right)$, there exists an open ball $\mathcal{B}$ centered at the point $(\alpha, \beta)$ such that the curve $\Upsilon$ splits this ball into two open subregions $\mathcal{B}^{\prime}$ and $\mathcal{B}^{\prime \prime}$ and in each of them the solution $x=0$ of the equation (10) is hyperbolic; for instance, if $\left(\alpha^{\prime}, \beta^{\prime}\right) \in B^{\prime}$, then the solution $x=0$ of the equation (10) is hyperbolic as $\alpha=\alpha^{\prime}$ and $\beta=\beta^{\prime}$ ).

Let us provide a scheme allowing one to identify approximately the boundaries of the hyperbolicity regions. The curve $\Upsilon$ defining the boundary of a hyperbolicity region can be described by some smooth function of $\beta=f(\alpha), \alpha=g(\beta)$ or it can be defined parametrically. We restrict ourselves by considering the first option, i.e., we construct the boundary of a hyperbolicity region for equation (10) as a curve described by a smooth function $\beta=f(\alpha)$ defined in some interval $\left|\alpha-\alpha_{0}\right|<\delta$ such that $f\left(\alpha_{0}\right)=\beta_{0}$. Namely, we construct the function $\beta=f(\alpha)$ as

$$
f(\alpha)=\beta_{0}+\beta_{1}\left(\alpha-\alpha_{0}\right)+\beta_{2}\left(\alpha-\alpha_{0}\right)^{2}+\eta\left(\alpha-\alpha_{0}\right),
$$

in which the coefficients $\beta_{1}$ and $\beta_{2}$ are to be determined, while the nonlinearity $\eta(\varepsilon)$ satisfies the relation $\eta(\varepsilon)=O\left(\varepsilon^{3}\right)$ as $\varepsilon \rightarrow 0$. In fact, the matter is to find the derivatives $f^{\prime}\left(\alpha_{0}\right)=\beta_{1}$ 
and $f^{\prime \prime}\left(\alpha_{0}\right)=2 \beta_{2}$. Because of this, we shall call the function

$$
\widehat{f}(\alpha)=\beta_{0}+\beta_{1}\left(\alpha-\alpha_{0}\right)+\beta_{2}\left(\alpha-\alpha_{0}\right)^{2}
$$

the asymptotic approximation of order 2 for the function $\beta=f(\alpha)$ at the point $\alpha=\alpha_{0}$. We proposed scheme can be developed also for constructing the asymptotic approximations for the function $\beta=f(\alpha)$ of higher order.

2.3.1. Preliminary transformations. Letting $\delta=\alpha-\alpha_{0}$ and substituting (12) into (10), we obtain the equation

$$
\frac{d x}{d t}=\left[A_{0}+\delta A_{1}\left(\beta_{1}, t\right)+\delta^{2} A_{2}\left(\beta_{1}, \beta_{2}, t\right)+A_{3}(t, \delta)\right] x+a(x, t, \alpha, \beta), \quad x \in \mathbb{R}^{N},
$$

where we denote

$$
A_{1}\left(\beta_{1}, t\right)=B_{1}(t)+\beta_{1} B_{2}(t), \quad A_{2}\left(\beta_{1}, \beta_{2}, t\right)=B_{11}(t)+\beta_{1} B_{12}(t)+\beta_{1}^{2} B_{22}(t)+\beta_{2} B_{2}(t),
$$

while the matrix $A_{3}(\delta, t)$ satisfies the relation $\left\|A_{3}(\delta, t)\right\|=O\left(\delta^{3}\right)$ as $\delta \rightarrow 0$ uniformly in $t$.

Hereinafter in all transformations, while denoting nonlinear terms (in equation (14), it is the function $a(x, t, \alpha, \beta)$ ), for the sake of brevity we keep the notations of the parameters $\alpha$ and $\beta$.

As in the case $2^{0}$, there exist non-zero vectors $e, g, e^{*}, g^{*} \in \mathbb{R}^{N}$ such that identities (7) and 8 hold true, in which $\omega_{0}=\frac{\pi k_{0}}{T}$. We let

$$
e_{1}=\frac{e+i g}{\sqrt{2}}, e_{2}=\frac{e-i g}{\sqrt{2}}, e_{1}^{*}=\frac{e^{*}+i g^{*}}{\sqrt{2}}, e_{2}^{*}=\frac{e^{*}-i g^{*}}{\sqrt{2}}
$$

By the definition, these functions are normalized by the identities

$$
\left(e_{1}, e_{1}^{*}\right)=\left(e_{2}, e_{2}^{*}\right)=1, \quad\left(e_{1}, e_{2}^{*}\right)=\left(e_{2}, e_{1}^{*}\right)=0 .
$$

The matrix $A_{0}$ and the transposed matrix $A_{0}^{*}$ have a pair of simple eigenvalues $\pm \omega_{0} i$. At that, the vectors $e_{1}, e_{1}^{*} \in \mathbb{C}^{N}$ defined by identities (15) are the eigenvectors of the operators $A_{0}$ and $A_{0}^{*}$ associated with the eigenvalues $\omega_{0} i$ and $-\omega_{0} i$, respectively. We introduce a linear operator in $\mathbb{C}^{N}$ by the formula $Q_{1} z=-2 \omega_{0} i P_{1} z$, where $P_{1} z=\left(z, e_{1}^{*}\right) e_{1} ; P_{1}$ is the spectral projector of a subspace in $\mathbb{C}^{N}$ on the one-dimensional eigenspace $E_{1}$ associated with the eigenvalues $\omega_{0} i$ of the operator $A_{0}$.

Equation 14 can be considered in the complex space $\mathbb{C}^{N}$. We make the change $y=e^{Q_{1} t} x$ in (14) with a non-degenerate $T$-periodic matrix $e^{Q_{1} t}$. By Lemma 1 this change preserves the topological type of the equilibrium point and transforms (14) to the form:

$$
\frac{d y}{d t}=\left[\tilde{A}+\delta \tilde{A}_{1}\left(\beta_{1}, t\right)+\delta^{2} \tilde{A}_{2}\left(\beta_{1}, \beta_{2}, t\right)+\tilde{A}_{3}(t, \delta)\right] y+\tilde{a}(y, t, \alpha, \beta), \quad y \in \mathbb{C}^{N}
$$

where

$$
\tilde{A}=A_{0}+Q_{1}, \quad \tilde{A}_{j}=e^{Q_{1} t} A_{j} e^{-Q_{1} t}(j=1,2,3), \quad \tilde{a}(y, t, \alpha, \beta)=e^{Q_{1} t} a\left(e^{-Q_{1} t} y, t, \alpha, \beta\right) .
$$

The operator $\tilde{A}$ has a semi-simple eigenvalue $-\omega_{0} i$ of multiplicity 2 , while its other eigenvalues have non-zero real parts. We denote by $E_{0}$ the spectral (two-dimensional) subspace of the operator $\tilde{A}$ associated with the eigenvalue $-\omega_{0} i$, and let $P_{0}: \mathbb{C}^{N} \rightarrow E_{0}$ be the spectral projector of the space $\mathbb{C}^{N}$ on the subspace $E_{0}$. The operator $P_{0}$ can be defined by the identity $P_{0} z=$ $\left(z, e_{1}^{*}\right) e_{1}+\left(z, e_{2}^{*}\right) e_{2}$. We let $P^{0}=I-P_{0}$ and $E^{0}=P^{0} \mathbb{C}^{N}$; the subspace $E^{0}$ is invariant for the operator $\tilde{A}$ and the operator $\tilde{A}: E^{0} \rightarrow E^{0}$ is hyperbolic. 
2.3.2. Calculation of the coefficient $\beta_{1}$. First we provide a scheme allowing us to calculate the coefficient $\beta_{1}$ for function (12). At the first step we consider the matrix equation

$$
\int_{0}^{T} e^{-\tilde{A} t} S e^{\tilde{A} t} d t=\int_{0}^{T} e^{-\tilde{A} t} \tilde{A}_{1}(\xi, t) e^{\tilde{A} t} d t
$$

depending on an auxiliary spectral parameter $\xi$, where the unknown is a square matrix $S$.

Lemma 2. Equation (17) has the unique solution $S=S(\xi)$ and the matrix $S(\xi)$ depends smoothly on $\xi$.

We let

$$
S_{0}(\xi)=P_{0} S(\xi) P_{0}
$$

and consider a two-dimensional linear operator $S_{0}(\xi): E_{0} \rightarrow E_{0}$. The matrix $S_{0}(\xi)$ of this operator is two-dimensional.

Theorem 5. Assume that there exist $\xi^{*}$ and $\delta_{0}>0$ such that the matrix $S_{0}\left(\xi^{*}\right)$ is nonhyperbolic and matrix $S_{0}(\xi)$ is hyperbolic as $\xi \in\left(\xi^{*}-\delta_{0}, \xi^{*}\right)$ and $\xi \in\left(\xi^{*}, \xi^{*}+\delta_{0}\right)$ with different topological type on thee intervals. Then there exists a boundary $\Upsilon$ of the hyperbolicity regions of the solution $x=0$ to equation (10) described by function (12), where $\beta_{1}=\xi^{*}$.

Let us consider a particular case, when the matrix $S_{0}(\xi)$ has a simple zero eigenvalue for some $\xi=\xi^{*}$.

Then it follows from the perturbation theory of linear operators [17] that for $\xi$ close to $\xi^{*}$, the matrix $S_{0}(\xi)$ has a simple real eigenvalue $\lambda(\xi)$ and the function $\lambda(\xi)$ depends smoothly on $\xi$ and the identity $\lambda\left(\xi^{*}\right)=0$ holds true.

Theorem 6. Let the matrix $S_{0}\left(\xi^{*}\right)$ has a simple eigenvalue $\lambda_{1}=0$, while its other eigenvalue $\lambda_{2}$ is a real non-zero number. Let $\lambda^{\prime}\left(\xi^{*}\right) \neq 0$. Then there exists a boundary $\Upsilon$ of the hyperbolicity regions of the solution $x=0$ to equation (10) described by function (12) with $\beta_{1}=\xi^{*}$.

Remark 1. If there exist $k$ different $\xi^{*}$ satisfying the assumptions of Theorem 5 or 6 , in the plane of the parameters $(\alpha, \beta)$, at least $k$ different curves $\Upsilon$ passthe point $\left(\alpha_{0}, \beta_{0}\right)$ and these curves are described by the functions of the form (12).

Let us note the following useful fact. In the space $\mathbb{C}^{N}$ we choose a basis formed by the vectors

$$
e_{1}, e_{2}, e_{3}, \ldots, e_{N}
$$

where $e_{1}, e_{2}$ are the vectors in $(15)$, while the vectors $e_{3}, \ldots, e_{N}$ form a basis in $E^{0}$. Then the matrix of the linear operator $\tilde{A}: \mathbb{C}^{N} \rightarrow \mathbb{C}^{N}$ is of the form:

$$
\tilde{A}=\left[\begin{array}{cc}
-\omega_{0} i I_{2} & O_{12} \\
O_{21} & A_{22}
\end{array}\right]
$$

where $I_{2}$ is the unit $2 \times 2$ matrix, $A_{22}$ is a square matrix of order $N-2$, while $O_{12}$ and $O_{21}$ are zero matrices of appropriate sizes. In this case the solution to equation (17), that is, the matrix $S(\xi)$ can be represented as

$$
S(\xi)=\left[\begin{array}{cc}
S_{0}(\xi) & S_{12}(\xi) \\
S_{21}(\xi) & S_{22}(\xi)
\end{array}\right]
$$

where $S_{0}(\xi)$ is matrix $(18), S_{22}(\xi)$ is a square matrix of size $N-2$, while $S_{12}(\xi)$ and $S_{21}(\xi)$ are rectangular matrices of appropriate sizes.

Together with the matrix $S(\xi)$, we shall also consider the matrix

$$
S_{1}(\xi)=\left[\begin{array}{cc}
S_{0}(\xi) & O_{12} \\
O_{21} & S_{22}(\xi)
\end{array}\right]
$$

For the sake of the convenience of the exposition, in what follows we suppose that as a basis in the space $\mathbb{C}^{N}$, vectors $(19)$ are chosen and therefore, identities 20 and $(21)$ hold true. 
2.3.3. Calculation of the coefficient $\beta_{2}$. Assume that the constant $\beta_{1}$ is found in accordance with Theorem 5 or 6 , that is, $\beta_{1}=\xi^{*}$. We proceed to calculating the coefficient $\beta_{2}$ of function (12). We consider system (16), where we let $\beta_{1}=\xi^{*}$, and it is convenient to redenote the unknown $\beta_{2}$ by $\nu$ :

$$
\frac{d y}{d t}=\left[\tilde{A}+\delta P_{1}(t)+\delta^{2} P_{2}(\nu, t)+\tilde{A}_{3}(t, \delta)\right] y+\tilde{a}(y, t, \alpha, \beta), \quad y \in \mathbb{C}^{N},
$$

where

$$
P_{1}(t)=\tilde{A}_{1}\left(\xi^{*}, t\right), \quad P_{2}(\nu, t)=\tilde{A}_{2}\left(\xi^{*}, \nu, t\right) .
$$

We let $S_{1}=S_{1}\left(\xi^{*}\right)$, where $S_{1}(\xi)$ is matrix (22). We then let

$$
H_{1}(t)=e^{\tilde{A} t}\left[\int_{0}^{t} e^{-\tilde{A} \tau}\left(P_{1}(\tau)-S_{1}\right) e^{\tilde{A} \tau} d \tau\right] e^{-\tilde{A} t} .
$$

Finally, we introduce the matrix

$$
F(\nu, t)=P_{2}(\nu, t)-H_{1}(t) P_{1}(t)+S_{1} H_{1}(t)
$$

and consider the matrix equation

$$
\int_{0}^{T} e^{-\tilde{A} t} Z e^{\tilde{A} t} d t=\int_{0}^{T} e^{-\tilde{A} t} F(\nu, t) e^{\tilde{A} t} d t
$$

depending on the parameter $\nu$, where the unknown is a square matrix $Z$.

Lemma 3. Equation (26) has the unique solution $Z=Z(\nu)$ which depends smoothly on $\nu$.

We let

$$
Z_{0}(\nu)=P_{0} Z(\nu) P_{0}
$$

and consider a two-dimensional linear operator $Z_{0}(\nu): E_{0} \rightarrow E_{0}$. The matrix $Z_{0}(\nu)$ of this operator is two-dimensional.

By identities 20 - 22), the matrix $Z(\nu)$ can be represented as

$$
Z(\nu)=\left[\begin{array}{ll}
Z_{0}(\nu) & Z_{12}(\nu) \\
Z_{21}(\nu) & Z_{22}(\nu)
\end{array}\right]
$$

We recall that the number $\xi^{*}$ is chosen by the condition that a two-dimensional matrix $S_{0}=S_{0}\left(\xi^{*}\right)$ is non-hyperbolic. Then, in an appropriate basis, the matrix $S_{0}$ has one of the following forms:

$$
\text { a) } \left.\left.\left[\begin{array}{cc}
0 & 0 \\
0 & \lambda_{2}
\end{array}\right], \quad b\right)\left[\begin{array}{cc}
i \omega_{1} & 0 \\
0 & -i \omega_{1}
\end{array}\right], \quad c\right)\left[\begin{array}{ll}
0 & 1 \\
0 & 0
\end{array}\right]
$$

where $\lambda_{2}$ is a non-zero real number and $\omega_{1} \geqslant 0$.

We restrict ourselves by considering the case, when the matrix $S_{0}\left(\xi^{*}\right)$ has the form a). Other cases can be considered in the same way in accordance with the provided below method by M. Roseau. In the case a) we write the two-dimensional matrix $Z_{0}(\xi)$ in the mentioned basis:

$$
Z_{0}(\nu)=\left[\begin{array}{ll}
z_{11}(\nu) & z_{12}(\nu) \\
z_{21}(\nu) & z_{22}(\nu)
\end{array}\right]
$$

Theorem 7. Suppose that under the assumptions of Theorem 6 the matrix $S_{0}\left(\xi^{*}\right)$ is of the form a). Let the function $\tau(\nu)=\operatorname{Re} z_{11}(\nu)$ satisfy the relations $\tau\left(\nu^{*}\right)=0$ and $\tau^{\prime}\left(\nu^{*}\right) \neq 0$ for some $\nu=\nu^{*}$. Then the boundary $\Upsilon$ of the hyperbolicity regions for the solution $x=0$ to the equation (10) is described by function (12), where $\beta_{1}=\xi^{*}$ and $\beta_{2}=\nu^{*}$. 
2.4. Linear and conservative systems. Consider the linear two-dimensional equation

$$
\frac{d x}{d t}=A(\alpha, \beta, t) x, \quad x \in \mathbb{R}^{2},
$$

where $A(\alpha, \beta, t)$ can be represented as $(11)$. Suppose that the assumptions of the case $3^{0}$ hold.

Since equation $(29)$ is a particular case of equation (10), Theorems 57 are true also for equation (29). However, linear equation (29) has its own features; in particular, the hyperbolicity properties are completely determined by the properties of the matrix $A(\alpha, \beta, t)$ that is not the case for nonlinear equation (10) in the critical cases, when equation (29) has multiplicators, whose absolute values are one. This is why the hyperbolicity regions for linear equation (29) and their boundaries can be determined under weaker conditions than Theorems 57.

In this subsection we consider the systems appearing in studying linear conservative systems. A linear autonomous system $x^{\prime}=A x$ is conservative if $\operatorname{tr} A=0$ (see, for instance, [2, 3]). In this case the matrix exponent $e^{A t}$ conserves the phase volume. A linear non-autonomous system $x^{\prime}=A(t) x$ with a $T$-periodic matrix $A(t)$ is conservative if the product of its multiplicators is equal to 1 . In this case the Poincaré mapping $U(T)$ of this system in time $T$ conserves the phase volume.

Assume that linear system (29) is conservative in aforementioned sense for all $\alpha$ and $\beta$. Reproducing the same constructions as for 10 in Case $3^{0}$ for this system, we obtain the matrix $S(\xi)$ being a solution to system (17).

Since considered system (29) is two-dimensional, the issue on its hyperbolicity regions is equivalent to the issue on its stability and instability regions.

Theorem 8. Assume that the matrix $S\left(\xi^{*}\right)$ has the zero double eigenvalue, Let there exists $\delta_{0}>0$ such that as $\xi \in\left(\xi^{*}-\delta_{0}, \xi^{*}+\delta_{0}\right)$, the matrix $S(\xi)$ has continuous branches of the eigenvalues $\lambda_{1}(\xi)$ and $\lambda_{2}(\xi)$ and at that,

- for $\xi \in\left(\xi^{*}, \xi^{*}+\delta_{0}\right)\left(\xi \in\left(\xi^{*}-\delta_{0}, \xi^{*}\right)\right)$ the eigenvalues $\lambda_{1}(\xi)$ and $\lambda_{2}(\xi)$ are real and $\lambda_{1}(\xi)<0$ and $\lambda_{2}(\xi)>0$

- for $\xi \in\left(\xi^{*}-\delta_{0}, \xi^{*}\right]\left(\xi \in\left[\xi^{*}, \xi^{*}+\delta_{0}\right)\right)$ the eigenvalues $\lambda_{1}(\xi)$ and $\lambda_{2}(\xi)$ are pure imaginary, namely, $\lambda_{1,2}= \pm i \omega(\xi)$, where $\omega\left(\xi^{*}\right)=0, \omega(\xi)>0$ as $\xi \neq \xi^{*}$.

Then there exists a boundary $\Upsilon$ of stability regions for equation (29) described by function (12), where $\beta_{1}=\xi^{*}$.

\section{AUXILIARY INFORMATION}

Here we provide some auxiliary information used in the proof of the statements of the present paper.

3.1. The method by M. Roseau. In the work we employ the method by M. Roseau proposed in [16] for linear differential equations

$$
z^{\prime}=\left[A+\varepsilon P_{1}(t)+\varepsilon^{2} P_{2}(t)+\ldots+\varepsilon^{k} P_{k}(t)+\varepsilon^{k+1} Q(t, \varepsilon)\right] z, \quad z \in \mathbb{C}^{N},
$$

with periodic coefficients and constant matrix $A$. The method proposes a way of passing to an equivalent equation of the form

$$
y^{\prime}=\left[A+\varepsilon S_{1}+\varepsilon^{2} S_{2}+\ldots+\varepsilon^{k} S_{k}+\varepsilon^{k+1} \tilde{Q}(t, \varepsilon)\right] y, \quad y \in \mathbb{C}^{N},
$$

where $S_{1}, S_{2}, \ldots, S_{k}$ are constant matrices and $\tilde{Q}(t, \varepsilon)$ is a continuous periodic matrix. Such passage allows one to solve the problem on stability of the mentioned linear system by constructing the eigenvalues of the constant matrix $A+\varepsilon S_{1}+\varepsilon^{2} S_{2}+\ldots+\varepsilon^{k} S_{k}$, for example, by the methods of the perturbation theory, [17]; in what follows we shall discuss this issue in more detaisl. In this section the method by M. Roseau is justified and developed for some nonlinear equations. 
3.1.1. Use of the first corrector. We first consider the differential equation

$$
\frac{d z}{d t}=\left[A+\varepsilon P(t)+\varepsilon^{2} Q(t, \varepsilon)\right] z+a(z, t, \varepsilon), \quad z \in \mathbb{C}^{N},
$$

depending on a small parameter $\varepsilon$, where $A$ is a constant matrix, $P(t)$ and $Q(t, \varepsilon)$ are continuous matrices, $a(z, t, \varepsilon)$ is a continuously differentiable in $x$ and continuous in $t$ and $\varepsilon$ vector function satisfying the condition $\|a(z, t, \varepsilon)\|=O\left(\|z\|^{2}\right)$ as $z \rightarrow 0$. At that, $P(t), Q(t, \varepsilon)$ and $a(z, t, \varepsilon)$ are $T$-periodic in $T$. The entries of matrices $A, P(t)$ and $Q(t, \varepsilon)$, as well as the vector function $a(z, t, \varepsilon)$ and parameter $\varepsilon$ can be both real and complex.

Let the matrix $A$ has one or several eigenvalues with zero real part, while other eigenvalues have negative real parts. In this case, for small $|\varepsilon|$, the stability properties of the solution $z=0$ to equation (32) depends on the multiplicators of a $T$-periodic matrix $A+\varepsilon P(t)$. However, these multiplicators can be found explicitly only in the simplest cases.

In order to study the stability of the solution $z=0$ to the nonlinear equation (32), we suggest to pass to the equivalent equation

$$
\frac{d y}{d t}=\left[A+\varepsilon S+\varepsilon^{2} \tilde{Q}(t, \varepsilon)\right] y+\tilde{a}(y, t, \varepsilon), \quad y \in \mathbb{C}^{N},
$$

where $S$ is a constant matrix. The passage can be made by the change

$$
y=(I-\varepsilon H(t)) z,
$$

where $H(t)$ is a some non-degenerate $T$-periodic matrix. The formula for $H(t)$ will be given later. Here we note that there exist infinitely many such matrices, and therefore, infinitely many matrices $S$ and that we do not need to know the matrix $H(t)$ in order to construct the matrix $S$.

Let us provide the scheme of constructing the matrix $S$. In order to do it, we introduce the following notion. We shall say that a square matrix $A$ is $T$-resonance if it has at least one pair of different eigenvalues $\lambda_{1}$ and $\lambda_{2}$ satisfying the relation: $\lambda_{1}-\lambda_{2}=\frac{2 \pi q i}{T}$ for some natural $q$. In particular, a real matrix $A$ is $T$-resonance if it has at least one pair of the eigenvalues of the form $\pm \frac{\pi q i}{T}$ for some natural $q$.

Let us consider the matrix equation

$$
\int_{0}^{T} e^{-A \tau} S e^{A \tau} d \tau=\int_{0}^{T} e^{-A \tau} P(\tau) e^{A \tau} d \tau
$$

for an unknown matrix $S$; here $A$ and $P(t)$ are the matrices in equation (32). The following statements take place [16].

Lemma 4. Equation (35) has the unique solution $S=S_{0}$ if and only if the matrix $A$ is not T-resonance.

Theorem 9. Assume that the matrix $A$ is not T-resonance. Then change (34), where

$$
H(t)=e^{A t}\left[\int_{0}^{t} e^{-A \tau}\left(P(\tau)-S_{0}\right) e^{A \tau} d \tau\right] e^{-A t},
$$

and $S_{0}$ is the unique equation to equation (35), transforms equation (32) to (33) with $S=S_{0}$.

Theorem 10. The matrices $S$ and $H(t)$, for which change (34) transforms equation (32) to (33), are not unique. Namely. let the matrix $A$ be not $T$-resonance and let $S_{0}$ be the solution to equation (35). Then change (34), where

$$
H(t)=e^{A t}\left[\int_{0}^{t} e^{-A \tau}(P(\tau)-S) e^{A \tau} d \tau+C_{0}\right] e^{-A t},
$$


and

$$
S=S_{0}-C_{0} A+A C_{0}
$$

transforms equation (32) to (33) for each constant matrix $C_{0}$.

Thus, if the matrix $A$ is not $T$-resonance, change (34) reduces equation (32) to (33). Here we note the following useful fact. By choosing a constant matrix $C_{0}$ in formula (38) we can get a "diagonal" matrix $S$ in equation (33). We provide an auxiliary statement in this direction.

Let the matrix $A$ has a semi-simple eigenvalue $\lambda_{0}$ of multiplicty $k$ and let $E_{0}$ be the associated eigenspace of the operator $A: \mathbb{C}^{N} \rightarrow \mathbb{C}^{N}$. Let $P_{0}$ be the spectral projector on the subspace $E_{0}$, that is, $P_{0}: \mathbb{C}^{N} \rightarrow E_{0}, A P_{0}=P_{0} A$ and $P_{0} x=x$ for each $x \in E_{0}$. We let $P^{0}=I-P_{0}$.

Lemma 5. The identity

$$
P_{0}\left(S_{0}-C_{0} A+A C_{0}\right) P_{0}=P_{0} S_{0} P_{0}
$$

holds true for each matrix $C_{0}$.

Thus, the matrix of the linear operator $P_{0}\left(S_{0}-C_{0} A+A C_{0}\right) P_{0}: E_{0} \rightarrow E_{0}$ is independent of the choice $C_{0}$.

Lemma 6. In identity (38), the matrix $C_{0}$ can be chosen so that the identities

$$
P_{0}\left(S_{0}-C_{0} A+A C_{0}\right) P^{0}=P^{0}\left(S_{0}-C_{0} A+A C_{0}\right) P_{0}=0, P^{0}\left(C_{0} A-A C_{0}\right) P^{0}=0
$$

are satisfied.

These identities can be checked by straightforward calculations.

It follows from these Lemmata that by a choice of $C_{0}$ we can get that matrix (38) has the form

$$
S=\left[\begin{array}{ll}
S_{11} & O_{12} \\
O_{21} & S_{22}
\end{array}\right]
$$

in an appropriate basis in the space $\mathbb{C}^{N}$, where $S_{11}$ is a square matrix of order $k$ of a linear operator $P_{0} S_{0} P_{0}: E_{0} \rightarrow E_{0}, S_{22}$ is a square matrix of order $N-k$, while $O_{12}$ and $O_{21}$ are zero rectangular matrices of appropriate sizes. At that, the matrices $S_{11}$ and $S_{22}$ coincide with corresponding diagonal blocks of the matrix $S_{0}$, which the solution to system (35) and moreover, the matrix $S_{11}$ is independent of the choice of $C_{0}$. In particular, if the matrix $A$ is simple (i.e., it has exactly $N$ different eigenvalues $\lambda_{1}, \lambda_{2}, \ldots, \lambda_{N}$ ), by choosing $C_{0}$ we can get that in the basis of the eigenvectors of the matrix $A$, matrix $(38)$ is of the form

$$
S=\left[\begin{array}{cccc}
s_{11} & 0 & \cdots & 0 \\
0 & s_{22} & \cdots & 0 \\
& & \ddots & \\
0 & 0 & \cdots & s_{N N}
\end{array}\right]
$$

at that, numbers $s_{j j}$ coincide with the corresponding diagonal elements of the matrix $S_{0}$, which is the solution to system (35).

Suppose now that the matrix $A$ is $T$-resonance. In this case we need to make a preliminary transformation $x=U(t) z$ with a $T$-periodic non-degenerate matrix $U(t)$, by means of which original system $(32)$ is reduced to the equivalent form

$$
\frac{d x}{d t}=\left[\tilde{A}+\varepsilon \tilde{P}(t)+\varepsilon^{2} \tilde{Q}(t, \varepsilon)\right] x+\tilde{a}(x, t, \varepsilon), \quad x \in \mathbb{C}^{N},
$$

in which the matrix $\tilde{A}$ is no longer $T$-resonance. Let, for instance, two different semi-simple eigenvalues $\lambda_{1}$ and $\lambda_{2}$ of the matrix $A$ satisfy the identity $\lambda_{1}-\lambda_{2}=\frac{2 \pi}{T} q i$ for some integer $q$. We denote by $E_{1}$ the spectral subspace of the operator $A$ associated with the eigenvalue $\lambda_{1}$, while by $P_{1}: \mathbb{C}^{N} \rightarrow E_{1}$ we denote the spectral projector. Letting $\tilde{P}_{1}=-\frac{2 \pi}{T} q i P_{1}$, in 32 we 
make the change $x=e^{\tilde{P}_{1} t} z$ with a $T$-periodic matrix $e^{\tilde{P}_{1} t}$. Then we obtain system $(39)$ of the required form, where $\tilde{A}=A+\tilde{P}_{1}$.

Thus, given an arbitrary matrix $A$, there exist infinitely many changes (34) transforming (32) to 33 .

3.1.2. Use of the next approximations. Now we consider a more general problem, namely, we consider equation (32) of the form:

$$
\frac{d z}{d t}=\left[A+\varepsilon P_{1}(t)+\varepsilon^{2} P_{2}(t)+\varepsilon^{3} Q(t, \varepsilon)\right] z+a(z, t, \varepsilon), \quad z \in \mathbb{C}^{N},
$$

where $A, Q(t, \varepsilon)$ and $a(z, t, \varepsilon)$ have the same meaning as in equation $(32), P_{1}(t)$ and $P_{2}(t)$ are continuous $T$-periodic matrices.

Let us provide the scheme of transforming to the equivalent equation

$$
\frac{d y}{d t}=\left[A+\varepsilon S_{1}+\varepsilon^{2} S_{2}+\varepsilon^{3} \tilde{Q}(t, \varepsilon)\right] y+\tilde{a}(y, t, \varepsilon), \quad y \in \mathbb{C}^{N},
$$

where $S_{1}$ and $S_{2}$ are constant matrices. The passage can be made by using the change

$$
y=\left(I-\varepsilon H_{1}(t)-\varepsilon^{2} H_{2}(t)\right) z,
$$

where $H_{1}(t)$ and $H_{2}(t)$ are some non-degenerate $T$-periodic matrices.

We assume that the matrix $A$ is not $T$-resonance; if this is not the case, it should be transformed as above. Let the matrices $S_{1}$ and $H_{1}(t)$ be determined by the above scheme. Namely, for the definiteness, let $S_{1}$ be the unique solution to an analogue of matrix equation (35):

$$
\int_{0}^{T} e^{-A \tau} S e^{A \tau} d \tau=\int_{0}^{T} e^{-A \tau} P_{1}(\tau) e^{A \tau} d \tau
$$

let $H_{1}(t)$ be defined in accordance with identity (36):

$$
H_{1}(t)=e^{A t}\left[\int_{0}^{t} e^{-A \tau}\left(P_{1}(\tau)-S_{1}\right) e^{A \tau} d \tau\right] e^{-A t},
$$

We let

$$
\tilde{P}_{2}(t)=P_{2}(t)-H_{1}(t) P_{1}(t)+S_{1} H_{1}(t)
$$

and consider the matrix equation

$$
\int_{0}^{T} e^{-A \tau} S e^{A \tau} d \tau=\int_{0}^{T} e^{-A \tau} \tilde{P}_{2}(\tau) e^{A \tau} d \tau
$$

with an unknown matrix $S$. Since the matrix $A$ is not $T$-resonance, equation (45) has the unique solution, which can be used as matrix $S_{2}$. Namely, we have the following theorem (see [16]).

Theorem 11. Let the matrix $A$ be not T-resonance, $S_{1}$ and $S_{2}$ be the unique solutions of equations (43) and (45), respectively. Then change (42), where $H_{1}(t)$ is matrix (44),

$$
H_{2}(t)=e^{A t}\left[\int_{0}^{t} e^{-A \tau}\left(\tilde{P}_{2}(\tau)-S_{2}\right) e^{A \tau} d \tau\right] e^{-A t},
$$

transforms equation (40) to (41).

As in Theorem 10, we can mention that matrices $S_{1}, S_{2}, H_{1}(t)$ and $H_{2}(t)$ are not uniquely defined. We also note that by the same scheme one can study the next approximations. 
3.1.3. A non-semi-simple eigenvalue. Thus, the method by M. Roseau allows us to reduce the issue on the stability of the zero solution to equation (30) to the similar issue for equation (31), which, in its turn, can be studied by constructing the eigenvalues of the constant matrix $A+\varepsilon S_{1}+\varepsilon^{2} S_{2}+\ldots+\varepsilon^{k} S_{k}$ depending on the small parameter $\varepsilon$. This problem can be solved by the methods of the perturbation theory of linear operators [17, 18, (see also the next subsection). In view of this, we mention that the methods of the perturbation theory are especially effective in the case, when the pure imaginary eigenvalues of the matrix $A$ are semi-simple. If the matrix $A$ has a non-semi-simple pure imaginary eigenvalue, the situation becomes much more complicated.

In this subsection we provide a way how to pass from a problem with non-semi-simple pure imaginary eigenvalues to an equivalent problem with semi-simple eigenvalue in the method by M. Roseau.

We restrict ourselves by consider the linear equation

$$
\frac{d x}{d t}=[A+\mu B(t)] x, \quad x \in \mathbb{C}^{N},
$$

depending on small parameter $\mu$, where $A$ and $B(t)$ are square matrices and $B(t+T) \equiv B(t)$. The matrices $A$ and $B(t)$ and the parameter $\mu$ in equation (47) can be real or complex.

Suppose that the matrix $A$ has a non-semi-simple double eigenvalue $\lambda_{0}$. Let $e$ and $g$ be an eigenvector and adjoint vector so that

$$
A e=\lambda_{0} e, \quad A g=\lambda_{0} g+e .
$$

The adjoint matrix $A^{*}$ has a non-semi-simple double eigenvalue $\overline{\lambda_{0}}$. Let $e^{*}$ and $g^{*}$ be an eigenvector and an adjoint vector of the matrix $A^{*}$. The vectors $e, g, e^{*}, g^{*}$ can be normalized as

$$
\left(e, e^{*}\right)=\left(g, g^{*}\right)=0,\left(e, g^{*}\right)=\left(g, e^{*}\right)=1 .
$$

We denote by $E_{0}$ the two-dimensional spectral subspace of the operator $A$ associated with the eigenvalue $\lambda_{0}$, while by $P_{0}: \mathbb{C}^{N} \rightarrow E_{0}$ we denote the spectral projector. This operator can be defined by the identity

$$
P_{0} x=\left(x, g^{*}\right) e+\left(x, e^{*}\right) g .
$$

We let $P^{0}=I-P_{0}$ and $E^{0}=P^{0} \mathbb{C}^{N}$. Then $E^{0}$ is an invariant subspace for the operator $A$ such that $\mathbb{C}^{N}=E_{0} \oplus E^{0}$, and $P^{0}$ is the spectral projector on $E^{0}$.

We let

$$
\begin{aligned}
& Q_{1} x=\left(x, g^{*}\right) e, \quad Q_{2} x=\left(x, e^{*}\right) g, \quad Q_{3} x=\left(x, e^{*}\right) e, \\
& Q_{0}(\varepsilon)=Q_{1}+\varepsilon Q_{2}, \quad Q(\varepsilon)=P^{0}+Q_{0}(\varepsilon),
\end{aligned}
$$

where $\varepsilon$ is a real or complex parameter. It is easy to prove the following lemma.

Lemma 7. The operator $Q(\varepsilon): \mathbb{C}^{N} \rightarrow \mathbb{C}^{N}$ is invertible as $\varepsilon \neq 0$ and

$$
(Q(\varepsilon))^{-1}=P^{0}+Q_{1}+\frac{1}{\varepsilon} Q_{2} .
$$

Assume that the parameters $\mu$ and $\varepsilon$ are related by the identity $\mu=\varepsilon^{2}$ so that $\varepsilon=\varepsilon(\mu)$ is one of the branches of the two-valued function $\varepsilon=\mu^{1 / 2}$. Then equation 47 becomes

$$
\frac{d x}{d t}=\left[A+\varepsilon^{2} B(t)\right] x, \quad x \in \mathbb{C}^{N} .
$$

As $\varepsilon \neq 0$, in equation 48 we make the change

$$
x=Q(\varepsilon) y \text { or } y=(Q(\varepsilon))^{-1} x .
$$

It is easy to confirm that change (49) transforms equation (48) to the form

$$
\frac{d y}{d t}=\left[A_{0}+\varepsilon B_{1}(t)+\varepsilon^{2} B_{2}(t)+\varepsilon^{3} B_{3}(t)\right] y, \quad y \in \mathbb{C}^{N},
$$


where

$$
\begin{aligned}
& A_{0}=A P^{0}+\lambda_{0} P_{0}, \\
& B_{1}(t)=Q_{3}+Q_{2} B(t)\left(P^{0}+Q_{1}\right), \\
& B_{2}(t)=\left(P^{0}+Q_{1}\right) B(t)\left(P^{0}+Q_{1}\right)+Q_{2} B(t) Q_{2}, \\
& B_{3}(t)=\left(P^{0}+Q_{1}\right) B(t) Q_{2} .
\end{aligned}
$$

By the definition, the operators $A: E_{0} \rightarrow E_{0}$ and $A_{0}: E_{0} \rightarrow E_{0}$ coincide. The operators $A: E_{0} \rightarrow E_{0}$ and $A_{0}: E_{0} \rightarrow E_{0}$ differ since $\lambda_{0}$ is a non-semi-simple double eigenvalue for the former and is a semi-simple double eigenvalue for the latter.

Thus, as $\varepsilon \neq 0$, equations (48) and (50) are equivalent in the sense that their solutions are related by identity (49). In particular, as $\varepsilon \neq 0$, the stability properties of these equations are same. At that, in equation (50), the eigenvalue $\lambda_{0}$ of the matrix $A_{0}$ is semi-simple.

Assume that $\operatorname{Re} \lambda_{0}=0$. For equation (50) we can employ the scheme by M. Roseau and in particular, we can pass to the equation of the form

$$
\frac{d z}{d t}=\left[A_{0}+\varepsilon S+\varepsilon^{2} B(t, \varepsilon)\right] y, \quad y \in \mathbb{C}^{N},
$$

where $S$ is a constant matrix. Studying the stability of the obtained matrix w.r.t. the constant matrix $A_{0}+\varepsilon S$, we obtain the solution for the problem on the stability of equation (48) as $\varepsilon \neq 0$, and therefore, of the original equation (47) as $\mu \neq 0$.

3.2. Formulae of perturbation theory. Assume that the matrix $A(\mu)$ depends continuously or smoothly on a scalar or vector parameter $\mu$ and the matrix $A\left(\mu_{0}\right)$ has a simple eigenvalue $\lambda_{0}$. Then for each $\mu$ close to $\mu_{0}$, the matrix $A(\mu)$ has the unique simple eigenvalue $\lambda(\mu)$ close to $\lambda_{0}$. We need to find this eigenvalue either analytically or approximately. Usually, such problems are solved by means of the perturbation theory of linear operators [17]. In this subsection we propose the formulae of the perturbation theory obtained in [18] by the method of the functionalization of the parameter. These formulae are employed in the proof of some statements in the present work. We shall assume that the matrix $A(\mu)$ is real and depends smoothly on the real parameter. In the below statements we use the notation $A^{\prime}=A^{\prime}\left(\mu_{0}\right)$.

Suppose first that as $\mu=\mu_{0}$, the matrix $A(\mu)$ has a simple real eigenvalue $\lambda_{0}$. Let $e_{0}$ and $g_{0}$ be the eigenvectors of the matrix $A_{0}=A\left(\mu_{0}\right)$ and the transposed matrix $A_{0}^{*}$ associated with the eigenvalue $\lambda_{0}$. The vectors $e_{0}$ and $g_{0}$ are assumed to be normalized by the identities $\left\|e_{0}\right\|=1$ and $\left(e_{0}, g_{0}\right)=1$.

Theorem 12. As $\mu$ is close to $\mu_{0}$, the matrix $A(\mu)$ has the unique simple real eigenvalue $\lambda(\mu)$ close to $\lambda_{0}$ and this eigenvalue is represented as

$$
\lambda(\mu)=\lambda_{0}+\gamma\left(\mu-\mu_{0}\right)+O\left(\left(\mu-\mu_{0}\right)^{2}\right) .
$$

where $\gamma=\left(A^{\prime} e_{0}, g_{0}\right)$.

In particular, formula (52) implies the identity $\lambda^{\prime}\left(\mu_{0}\right)=\left(A^{\prime} e_{0}, g_{0}\right)$.

Assume that the matrix $A_{0}=A\left(\mu_{0}\right)$ has a pair of simple eigenvalues $\pm \omega_{0} i$, where $\omega_{0}>0$. Then for small $\left|\mu-\mu_{0}\right|$, the matrix $A(\mu)$ has a pair of simple eigenvalues $\lambda(\mu)=\alpha(\mu) \pm i \omega(\mu)$, where $\alpha\left(\mu_{0}\right)=0$ and $\omega\left(\mu_{0}\right)=\omega_{0}$. There exist non-zero eigenvectors $e, g, e^{*}, g^{*}$ such that identities (7) and (8) hold true.

Theorem 13. As $\mu$ is close to $\mu_{0}$, the real and imaginary parts of the eigenvalue $\lambda(\mu)=$ $\alpha(\mu) \pm i \omega(\mu)$ of the matrix $A(\mu)$ satisfy the representation

$$
\begin{aligned}
& \alpha(\mu)=\frac{\gamma_{1}}{2}\left(\mu-\mu_{0}\right)+O\left(\left(\mu-\mu_{0}\right)^{2}\right), \\
& \omega(\mu)=\omega_{0}-\frac{\gamma_{2}}{2}\left(\mu-\mu_{0}\right)+O\left(\left(\mu-\mu_{0}\right)^{2}\right),
\end{aligned}
$$


where

$$
\gamma_{1}=\left(A^{\prime} e, e^{*}\right)+\left(A^{\prime} g, g^{*}\right), \quad \gamma_{2}=\left(A^{\prime} e, g^{*}\right)-\left(A^{\prime} g, e^{*}\right) .
$$

\section{Application: Mathieu equation}

As an application, we consider the Mathieu equation

$$
u^{\prime \prime}+(\alpha+\beta \cos 2 t) u=0,
$$

where $\alpha$ and $\beta$ are real parameters. A huge amount of works were devoted to studying various problems related to the Mathieu equation, see, for instance, [6, 13]). Here one of the main problems is the stability of the solutions, in particular, the issue on constructing the stability regions in the plane of the parameters $(\alpha, \beta)$. It is known that if $|\beta|$ is small and $\alpha<0$, then the solution $u=0$ to equation (55) is unstable; if $\alpha \geqslant 0$, then, as a rule, for small $|\beta|$ the solution $u=0$ is stable except the vicinities of the points $\left(n^{2}, 0\right)$ in the space $(\alpha, \beta)$. Namely, the instability regions wedge themselves as thin tongues in the points $\left(n^{2}, 0\right)$.

As an example, we provide the scheme of constructing one of such tongues for $n=1$. In order to do it, we shall consider the problem on studying the stability of the solution $u=0$ to equation (55) for the values of $\alpha$ and $\beta$ close to $\alpha_{0}=1$ and $\beta_{0}=0$, respectively.

By the standard change $z_{1}=u, z_{2}=u^{\prime}$ we transform equation (55) to a system of the form (10), namely, to the linear system

$$
x^{\prime}=A(\alpha, \beta, t) x, \quad x \in \mathbb{R}^{2},
$$

where

$$
A(\alpha, \beta, t)=\left[\begin{array}{cc}
0 & 1 \\
-(\alpha+\beta \cos 2 t) & 0
\end{array}\right] .
$$

In this example $T=\pi$ and the identity

$$
A_{0}=A(1,0, t)=\left[\begin{array}{cc}
0 & 1 \\
-1 & 0
\end{array}\right]
$$

holds true. The eigenvalue of this matrix are equal to $\pm i$, that is, in the considered example we have $\omega_{0}=1$. Finally, since $\omega_{0}=\frac{\pi k_{0}}{T}$ as $k_{0}=1$, for the equation 56 , case $3^{0}$ holds true as $\alpha_{0}=1$ and $\beta_{0}=0$.

We seek a curve $\Upsilon_{0}$ bordering the stability and instability region of equation (56) and passing through the point $(1,0)$ in the plane of the parameters $(\alpha, \beta)$ as function $(12)$ :

$$
\beta=\beta_{1} \delta+\beta_{2} \delta^{2}+\eta(\delta)
$$

where $\delta=\alpha-1$, while $\beta_{1}$ and $\beta_{2}$ are coefficients to be determined.

4.0.1. Preliminary transformations. First we calculate the coefficient $\beta_{1}$ in accordance with the above scheme. In our example equation (14) has the form

$$
\frac{d x}{d t}=\left[A_{0}+\delta A_{1}\left(\beta_{1}, t\right)+\delta^{2} A_{2}\left(\beta_{2}, t\right)+A_{3}(t, \delta)\right] x, \quad x \in \mathbb{R}^{2},
$$

where $A_{0}$ is matrix (58),

$$
A_{1}\left(\beta_{1}, t\right)=-\left(1+\beta_{1} \cos 2 t\right)\left[\begin{array}{ll}
0 & 0 \\
1 & 0
\end{array}\right], \quad A_{2}\left(\beta_{2}, t\right)=-\frac{1}{2} \beta_{2} \cos 2 t\left[\begin{array}{ll}
0 & 0 \\
1 & 0
\end{array}\right] .
$$

Before we transform equation $(60)$ to $(16)$, for the convenience of calculations, we reduce the matrix $A_{0}$ to the diagonal form choosing its eigenvectors as a basis $\mathbb{C}^{2}$. In other words, in 60 we make the change $x=Q z$, where $Q=\left[\begin{array}{ll}1 & i \\ i & 1\end{array}\right]$. Then equation 60 has the form

$$
\frac{d z}{d t}=\left[A_{00}+\delta A_{10}\left(\beta_{1}, t\right)+\delta^{2} A_{20}\left(\beta_{2}, t\right)+A_{30}(t, \delta)\right] z, \quad z \in \mathbb{C}^{2},
$$


where

$$
\begin{aligned}
& A_{00}=\left[\begin{array}{rr}
i & 0 \\
0 & -i
\end{array}\right], \quad A_{10}\left(\beta_{1}, t\right)=-\frac{1}{2}\left(1+\beta_{1} \cos 2 t\right)\left[\begin{array}{cc}
-i & 1 \\
1 & i
\end{array}\right], \\
& A_{20}\left(\beta_{2}, t\right)=-\frac{1}{4} \beta_{2} \cos 2 t\left[\begin{array}{cc}
-i & 1 \\
1 & i
\end{array}\right] .
\end{aligned}
$$

Let us transform obtained equation (61) to (16). In order to do it, we need to make the change $y=e^{Q_{1} t} z$ in 61 , where the operator $Q_{1}$ is determined by the identity $Q_{1} x=-2 \omega_{0} i\left(x, e_{1}^{*}\right) e_{1}$. In our case we have $e_{1}=e_{1}^{*}=\left[\begin{array}{l}1 \\ 0\end{array}\right]$ and, therefore, the matrix of the operators $Q_{1}$ is determined by the identity $Q_{1}=\left[\begin{array}{cc}-2 i & 0 \\ 0 & 0\end{array}\right]$. Then the change $y=e^{Q_{1} t} z$ reduces equation 61 to the form (16):

$$
\frac{d y}{d t}=\left[\tilde{A}+\delta \tilde{A}_{1}\left(\beta_{1}, t\right)+\delta^{2} \tilde{A}_{2}\left(\beta_{2}, t\right)+\tilde{A}_{3}(t, \delta)\right] y, \quad y \in C^{2}
$$

where

$$
\begin{aligned}
& \tilde{A}=-i\left[\begin{array}{ll}
1 & 0 \\
0 & 1
\end{array}\right], \quad \tilde{A}_{1}\left(\beta_{1}, t\right)=-\frac{1}{2}\left(1+\beta_{1} \cos 2 t\right)\left[\begin{array}{rr}
-i & e^{-2 i t} \\
e^{2 i t} & i
\end{array}\right], \\
& \tilde{A}_{2}\left(\beta_{2}, t\right)=-\frac{1}{2} \beta_{2} \cos 2 t\left[\begin{array}{cr}
-i & e^{-2 i t} \\
e^{2 i t} & i
\end{array}\right] .
\end{aligned}
$$

4.0.2. Calculation of coefficient $\beta_{1}$. Finally, we proceed to considering matrix equation (17). Since $\tilde{A}=-i I$, where $I$ is the unit matrix, here equation 17 is of the form

$$
\int_{0}^{\pi} S d t=\int_{0}^{\pi} \tilde{A}_{1}(\xi, t) d t
$$

and its solution is the matrix

$$
S(\xi)=\frac{1}{4}\left[\begin{array}{cc}
2 i & -\xi \\
-\xi & -2 i
\end{array}\right]
$$

Since the eigenvalue of the matrix $S(\xi)$ solve the equation $\lambda^{2}=\frac{\xi^{2}-4}{16}$, the assumptions of Theorem 8 are satisfied as $\xi= \pm 2$. Therefore, the coefficient $\beta_{1}$ in representation of function (59) can have two possible values: $\beta_{1}=2$ and $\beta_{1}=-2$. These values determine the slope $\varphi= \pm \arctan 2$ of the tangents to the boundaries of the stability and instability regions for Mathieu equation (55) at the point $(1,0)$ in the space of parameters $(\alpha, \beta)$.

4.0.3. Calculation of coefficient $\beta_{2}$. Let us find the coefficient $\beta_{2}$ in the representation of function (59). For the definiteness we let $\beta_{1}=2$. We also let

$$
S_{1}=S(2)=\frac{1}{2}\left[\begin{array}{cc}
i & -1 \\
-1 & -i
\end{array}\right] .
$$

Then in system (23) we have

$$
\begin{aligned}
& P_{1}(t)=\tilde{A}_{1}(2, t)=-\frac{1}{2}(1+2 \cos 2 t)\left[\begin{array}{rr}
-i & e^{-2 i t} \\
e^{2 i t} & i
\end{array}\right], \\
& P_{2}(\nu, t)=\tilde{A}_{2}(\nu, t)=-\frac{1}{2} \nu \cos 2 t\left[\begin{array}{rr}
-i & e^{-2 i t} \\
e^{2 i t} & i
\end{array}\right] .
\end{aligned}
$$

Since $\tilde{A}=-i I$, matrix 24 is equal to

$$
H_{1}(t)=\int_{0}^{t}\left(P_{1}(\tau)-S_{1}\right) d \tau=-\frac{1}{8}\left[\begin{array}{cr}
-4 i \sin 2 t & 2 i e^{-2 i t}+i e^{-4 i t}-3 i \\
-2 i e^{2 i t}-i e^{4 i t}+3 i & 4 i \sin 2 t
\end{array}\right] .
$$


Then matrix (25) is equal to

$$
F(\nu, t)=P_{2}(\nu, t)-H_{1}(t) P_{1}(t)+S_{1} H_{1}(t)=\left[\begin{array}{ll}
f_{11}(\nu, t) & f_{12}(\nu, t) \\
f_{21}(\nu, t) & f_{22}(\nu, t)
\end{array}\right],
$$

where

$$
\begin{aligned}
& f_{11}(\nu, t)=\frac{1}{2} \nu i \cos 2 t-\frac{1}{16}\left(-2 i+4 i \cos 2 t-2 i \cos ^{2} 2 t+2 \sin 2 t-\sin 4 t\right), \\
& f_{12}(\nu, t)=-\frac{1}{2} \nu e^{-2 i t} \cos 2 t-\frac{1}{16}\left(2-2 e^{-4 i t}-2 i e^{-2 i t} \sin 4 t\right), \\
& f_{21}(\nu, t)=f_{12}(\nu,-t), \\
& f_{22}(\nu, t)=-\frac{1}{2} \nu i \cos 2 t-\frac{1}{16}\left(2 i-4 i \cos 2 t+2 i \cos ^{2} 2 t+2 \sin 2 t-\sin 4 t\right) .
\end{aligned}
$$

Finally, a solution to matrix equation 26 is of the form

$$
Z(\nu)=\frac{1}{\pi} \int_{0}^{\pi} F(\nu, t) d t=\left[\begin{array}{cr}
\frac{3}{16} i & -\frac{1}{4} \nu-\frac{1}{8} \\
-\frac{1}{4} \nu-\frac{1}{8} & -\frac{3}{16} i
\end{array}\right] .
$$

Thus, in accordance with the method by M. Roseau, the stability of system (23) is equivalent to the stability of system

$$
\frac{d y}{d t}=\left[-i I+\delta S_{1}+\delta^{2} Z(\nu)+\tilde{A}_{3}(t, \delta)\right] y, \quad y \in \mathbb{C}^{2} .
$$

We note that since matrix $S_{1}$ defined by identity (63) has a non-semi-simple double zero eigenvalue, we have case c) described in (28).

We make a non-degenerate periodic change $x=e^{i t} y$ in system (66); then we obtain the system

$$
\frac{d x}{d t}=\left[\delta S_{1}+\delta^{2} Z(\nu)+\tilde{A}_{3}(t, \delta)\right] x, \quad x \in \mathbb{C}^{2} .
$$

For the sake of convenient calculations, we reduce the matrix $S_{1}$ to the canonical form taking its eigenvector and adjoint vector as a basis in $\mathbb{C}^{2}$. In other words, in (67), we make the change $x=Q z$, where $Q=\left[\begin{array}{cc}1 & -i \\ i & -1\end{array}\right]$. Then we arrive at the system:

$$
\frac{d z}{d t}=\left[\delta \tilde{S}_{1}+\delta^{2} \tilde{Z}+Q^{-1} \tilde{A}_{3}(t, \delta) Q\right] z, \quad z \in C^{2},
$$

where $\tilde{S}_{1}=Q^{-1} S_{1} Q=\left[\begin{array}{ll}0 & 1 \\ 0 & 0\end{array}\right], \quad \tilde{Z}=Q^{-1} Z Q=\left[\begin{array}{cr}0 & \frac{1}{4} \nu+\frac{5}{16} \\ \frac{1}{4} \nu-\frac{1}{16} & 0\end{array}\right]$.

By the scheme described in Subsection 3.1.3 we let

$$
\begin{gathered}
e=\left[\begin{array}{l}
1 \\
0
\end{array}\right], g=\left[\begin{array}{l}
0 \\
1
\end{array}\right], \quad e^{*}=\left[\begin{array}{l}
0 \\
1
\end{array}\right], g^{*}=\left[\begin{array}{l}
1 \\
0
\end{array}\right], \\
Q_{1} x=\left(x, g^{*}\right) e, \quad Q_{2} x=\left(x, e^{*}\right) g, \quad Q_{3} x=\left(x, e^{*}\right) e, \quad Q_{0}(\varepsilon)=Q_{1}+\varepsilon Q_{2},
\end{gathered}
$$

where $\varepsilon$ is a small parameter. As $\varepsilon \neq 0$, the operator $Q_{0}(\varepsilon)$ is invertible and

$$
\left(Q_{0}(\varepsilon)\right)^{-1}=Q_{1}+\frac{1}{\varepsilon} Q_{2} \text {. }
$$

Since $\beta_{1}=2$, without loss of generality, in $(59)$ we can assume that $\delta>0$. From $\delta$, we pass to a new real parameter $\varepsilon$ related with $\delta$ by the identity $\varepsilon=\sqrt{\delta}$. And for $\varepsilon>0$ in equation (68) we make the change $z=Q_{0}(\varepsilon) x$. Then obtain the equation:

$$
\frac{d x}{d t}=\left[\varepsilon^{3} \widehat{S}_{1}+\varepsilon^{5} \widehat{Z}+\left(Q_{0}(\varepsilon)\right)^{-1} Q^{-1} \tilde{A}_{3}(t, \varepsilon) Q Q_{0}(\varepsilon)\right] x, \quad x \in \mathbb{C}^{2},
$$




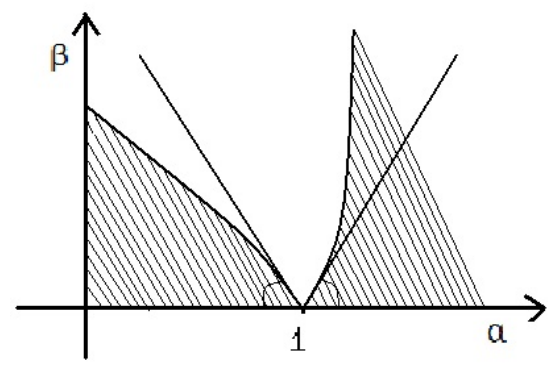

FiguRE 2. Stability regions of the Mathieu equation.

where

$$
\widehat{S}_{1}=\left[\begin{array}{cc}
0 & 1 \\
\frac{1}{4} \nu-\frac{1}{16} & 0
\end{array}\right], \quad \widehat{Z}=\left[\begin{array}{lr}
0 & \frac{1}{4} \nu+\frac{5}{16} \\
0 & 0
\end{array}\right] .
$$

The matrix $\widehat{S}_{1}$ is stable as $\nu<1 / 4$ and unstable as $\nu>1 / 4$. Therefore, in accordance with an analogue of Theorem 7 we can let $\nu^{*}=\frac{1}{4}$.

In the same way, if we take $\beta_{1}=-2$ in the representation of function (59), we obtain $\nu^{*}=-\frac{1}{4}$.

Thus, the desired boundaries of the stability regions are described by functions (59) of the form:

$$
\begin{aligned}
& \beta=2(\alpha-1)+\frac{1}{4}(\alpha-1)^{2}+\eta_{1}(\alpha-1), \\
& \beta=-2(\alpha-1)-\frac{1}{4}(\alpha-1)^{2}+\eta_{2}(\alpha-1),
\end{aligned}
$$

where the functions $\eta_{j}(\delta)$ satisfy the relations $\eta_{j}(\delta)=o\left(\delta^{2}\right)$ as $\delta \rightarrow 0$. In Figure, the curves bordering the stability and instability regions of the Mathieu equation are shown; the stability regions are shaded.

\section{Proofs of the MAin STATEMEnts}

5.1. Proof of Lemma 1. We consider the matrix Cauchy problems:

$$
\left\{\begin{array} { l } 
{ X ^ { \prime } = A ( t ) X , } \\
{ X ( 0 ) = I , }
\end{array} \quad \left\{\begin{array}{l}
Y^{\prime}=B(t) Y, \\
Y(0)=I,
\end{array}\right.\right.
$$

where $A(t)$ and $B(t)$ are the matrices in (3) and (4). Let $X(t)$ and $Y(t)$ be the solutions of these problems. Then the matrices $X(T)$ and $Y(T)$ are the monodromy matrices for the linear systems $x^{\prime}=A(t) x$ and $y^{\prime}=B(t) y$, that is, the eigenvalues of these matrices, the multiplicators, determine the topological types of the zero equilibrium points of systems (3) and (4). The proof of Lemma 1 will be completed if we show that the matrices $X(T)$ and $Y(T)$ are similar.

In order to do it, we consider one more Cauchy problem

$$
\left\{\begin{array}{l}
Z^{\prime}=B(t) Z, \\
Z(0)=U_{0},
\end{array}\right.
$$

where $U_{0}=U(0)$, and $U(t)$ is the matrix in the formula $y=U(t) x$ describing the transform of (3) to (4). Let $Z(t)$ be the solution to problem (69). It is easy to see that the matrices $X(t)$ and $Z(t)$ are related by the identity $Z(t)=U(t) X(t)$, and the matrices $Y(t)$ and $Z(t)$ are related by the identity $Y(t)=Z(t) U_{0}^{-1}$. It yields the identity $Y(T)=U_{0} X(T) U_{0}^{-1}$ that completes the proof. 
5.2. Proof of Theorem 1. Equation (5) has the form (32). Since the matrix $A_{0}$ is real and its topological type is equal to $\left(p_{-}, 1, p_{+}\right)$, the matrix $A_{0}$ is not $T$-resonance. This is why we can apply the scheme by $\mathrm{M}$. Roseau, namely, Theorem 9 can be applied to equation (5). In other words, there exists a change $y=(I-\varepsilon H(t)) x$ with a non-degenerate and $T$-periodic matrix $H(t)$ reducing (5) to the form (33):

$$
\frac{d y}{d t}=\left[A_{0}+\mu S_{0}+\mu^{2} \tilde{O}(\mu, t)\right] y+\tilde{a}(y, t, \mu), \quad y \in \mathbb{C}^{N}
$$

where the matrix $S=S_{0}$ is the unique solution to the equation

$$
\int_{0}^{T} e^{-A_{0} \tau} S e^{A_{0} \tau} d \tau=\int_{0}^{T} e^{-A_{0} \tau} A_{1}(\tau) e^{A_{0} \tau} d \tau
$$

According to Theorem 12 , the matrix $\tilde{A}(\mu)=A_{0}+\mu S_{0}$ has the unique eigenvalue close to zero for small $|\mu|$ and it satisfies the representation

$$
\lambda(\mu)=\left(S_{0} e_{0}, g_{0}\right) \mu+O\left(\mu^{2}\right) .
$$

Here $e_{0}$ and $g_{0}$ are the eigenvectors of the matrix $A_{0}$ and the transposed matrix $A_{0}^{*}$ associated with the zero eigenvalue.

Letting $S=S_{0}$ in (71) and multiplying both sides of the obtained identity first by the vector $e_{0}$ and calculating the scalar product with the vector $g_{0}$, we obtain the identity:

$$
\left(S e_{0}, g_{0}\right)=\frac{1}{T} \int_{0}^{T}\left(A_{1}(\tau) e_{0}, g_{0}\right) d \tau=\frac{1}{T} \lambda_{1},
$$

where $\lambda_{1}$ is number (6).

By Lemma 1, the topological types of the zero equilibrium points of systems (5) and (70) have the same topological type for small $|\mu|$. For small $\mu$ the topological type of the zero equilibrium point of system (70) is determined by the properties of the matrix $\tilde{A}(\mu)=A_{0}+\mu S_{0}$. But by the assumption of the theorem the topological type of matrix $A_{0}$ is equal to $\left(p_{-}, 1, p_{+}\right)$. This is why it follows from the identities $(72)$ and $(73)$ that for small $|\mu|$ the topological type of the zero equilibrium point of system $\sqrt{70})$ is equal to $\left(1+p_{-}, 0, p_{+}\right)$if $\mu \lambda_{1}<0$ and it is equal to $\left(p_{-}, 0,1+p_{+}\right)$if $\mu \lambda_{1}>0$. The proof is complete.

5.3. Proof of Theorem 3. The proof of Theorem 3 follows the same scheme as the proof of Theorem 1. At that we use asymptotic formulae (53) and (54) from Theorem 13 .

5.4. Proof of Lemma 2. The proof of the existence and uniqueness of the solution $S(\xi)$ to equation (17) can be found in [16]. The fact that the function $S(\xi)$ is smooth is implied by the assumption that in equation $\sqrt{10})$, the entries of the matrix $A(\alpha, \beta, t)$ are twice continuously differentiable in $\alpha$ and $\beta$.

5.5. Proofs of Theorems 5 and 6. We restrict ourselves by showing the scheme of the proof of Theorem 6. The general statement (i.e., Theorem 5) can be proved by the same scheme but the proof is more cumbersome since we have to consider various cases; one of these cases is contained in Theorem 6. For the sake of simplicity we assume that equation (10) is two-dimensional, i.e., $N=2$.

We consider the linear system corresponding to 100 :

$$
\frac{d x}{d t}=A(\alpha, \beta, t) x, \quad x \in \mathbb{R}^{2},
$$

the properties of its Floquet exponents determine the hyperbolicity regions and their boundaries for the zero solution to equation (10). We recall the assumption that the matrix $A_{0}=A\left(\alpha_{0}, \beta_{0}, t\right)$ is independent of $t$ and the matrix $A_{0}$ has a pair of simple eigenvalues $\pm i \omega_{0}$, where $\omega_{0}=\frac{\pi k_{0}}{T}$ for some natural $k_{0}$. 
By the perturbation theory of linear operators (see, for instance, [17]), system (74) has two Floquet exponents $\lambda_{1}(\alpha, \beta)$ and $\lambda_{2}(\alpha, \beta)$ for $(\alpha, \beta)$ close to $\left(\alpha_{0}, \beta_{0}\right)$ and the real parts of these exponents are determined uniquely. We let $u_{j}(\alpha, \beta)=\operatorname{Re} \lambda_{j}(\alpha, \beta), j=1,2$; then $u_{j}\left(\alpha_{0}, \beta_{0}\right)=0$.

Employing the condition $\lambda^{\prime}\left(\xi^{*}\right) \neq 0$ of Theorem 6 and the fact that by Lemma 1 , the nondegenerate $T$-periodic transformations of equation (10) made in Subsection 2.3 conserves the topological type of its zero solution, one can show that one of the functions $u_{j}(\alpha, \beta)$ satisfies the identity $u_{j \beta}^{\prime}\left(\alpha_{0}, \beta_{0}\right)=\lambda^{\prime}\left(\xi^{*}\right)$. For the sake of definiteness, we assume that this identity holds for $j=1$.

We consider the equation

$$
u_{1}(\alpha, \beta)=0 .
$$

By the implicit function theorem, equation (75) determines the unique function $\beta=f(\alpha)$ defined in some interval $\left(\alpha_{0}-\delta_{0}, \alpha_{0}+\delta_{0}\right)$ such that $f\left(\alpha_{0}\right)=\beta_{0}$. At that, function $\beta=f(\alpha)$ is continuously differentiable. By a straightforward calculation we can make sure that $f^{\prime}\left(\alpha_{0}\right)=\xi^{*}$. The curve $\Upsilon$ described by the function $\beta=f(\alpha)$ is the sought boundary of the hyperbolicity region for the zero solution to equation (10). The proof is complete.

5.6. Proof of Theorem 7. The proof of Theorem 7 follows the same scheme as for Theorem 6 .

5.7. Proof of Theorem 8. Letting for simplicity of the notations $\alpha_{0}=0$ and $\beta_{0}=0$, we rewrite equation 29 as

$$
\frac{d x}{d t}=\left[A_{0}+\alpha A_{1}(t)+\beta B_{1}(t)+B_{3}(\alpha, \beta, t)\right] x, \quad x \in \mathbb{R}^{2},
$$

where the matrix $B_{3}(\alpha, \beta, t)$ satisfies the relation: $\max _{t}\left\|B_{3}(\alpha, \beta, t)\right\|=O\left(\alpha^{2}+\beta^{2}\right)$ as $(\alpha, \beta) \rightarrow$ $(0,0)$.

By transformations similar to that made for equation (10), we transform linear equation (76) to 16$)$ :

and by the change $z=e^{i \omega_{0} t} y$ it is reduced to

$$
\frac{d y}{d t}=\left[\tilde{A}+\alpha \tilde{A}_{1}(t)+\beta \tilde{B}_{1}(t)+\tilde{B}_{3}(\alpha, \beta, t)\right] y,
$$

$$
\frac{d z}{d t}=\left[\alpha \tilde{A}_{1}(t)+\beta \tilde{B}_{1}(t)+\tilde{B}_{3}(\alpha, \beta, t)\right] z .
$$

We denote by $X(\alpha, \beta)$ the monodromy matrix of system $(77)$. Then $X(0,0)=I$, where $I$ is the unit matrix. The boundary of the stability region for equation (29) is determined by the equation

$$
\operatorname{det}(X(\alpha, \beta)-I)=0 .
$$

To complete the proof of Theorem 8 , it remains to observe that under its assumptions, equation (78) has a solution of the form $\beta=\xi^{*} \alpha+O\left(\alpha^{2}\right)$ determining the stability boundary for equation (76).

\section{BIBLIOGRAPHY}

1. J. Guckenheimer, P.J. Holmes. Nonlinear oscillations, dynamical systems, and bifurcations of vector fields. Springer, New York (1983).

2. V.I. Arnold. Geometrical methods in the theory of ordinary differential equations. Regularn. Khaotichesk. Dinam., Izhevsk (2000). [A Series of Comprehensive Studies in Mathematics. 250. Springer, New York (1988)]

3. A.B. Katok, B. Hasselblatt. Introduction to the modern theory of dynamical systems. Cambridge Univ. Press, Cambridge (1997).

4. L.P. Shil'nikov, A.L. Shil'nikov, D.V. Turaev, L. Chua. Methods of qualitative theory in nonlinear dynamics. II. Institut Kompyuter. Issledov., Moscow-Izhevsk (2009). [World Scientific Series on Nonlinear Science. Series A 5. World Scientific Publishing, Singapore (2001).] 
5. H.D. Chiang, L.F.C. Alberto. Stability regions of nonlinear dynamical systems: theory, estimation, and applications. Cambridge Univ. Press, Cambridge (2015).

6. B.P. Demidovich. Lectures on mathematical stability theory. Nauka, Moscow (1967). (in Russian).

7. V.A. Yakubovich, V.M. Starzhinskij. Linear differential equations with periodic coefficients. Nauka, Moscow (1972). [John Wiley \& Sons, New York (1975).]

8. M. Loccufier, E. Noldus. A new trajectory reversing method for estimating stability regions of autonomous nonlinear systems // Nonlinear Dynamics. 21:3, 265-288 (2000).

9. H.D. Chiang, M.W. Hirsch, F.F. Wu. Stability region of nonlinear autonomous dynamical systems. // IEEE Trans. Autom. Control. 33:1, 16-27 (1988).

10. F.M. Amaral, L.F.C. Alberto. Stability boundary characterization of nonlinear autonomous dynamical systems in the presence of a saddle-node equilibrium point // Tend. Mat. Apl. Comput. 13:2, 143-154 (2012).

11. M.A. Krasnosel'skij, N.A. Kuznetsov, M.G. Yumagulov. Parameter functionability and asymptotic behavior of cycles in Hopf's bifurcation // Avtomatika i Telemekhanika. 11, 22-28 (1996). [Autom. Remote Control 57:11, 1548-1553 (1996).]

12. M.A. Krasnosel'skij, N.A. Kuznetsov, M.G. Yumagulov. An operator method for investigating the stability of cycles in Hopf bifurcation // Avtomatika i Telemekhanika. 12, 24-30 (1996). [Autom. Remote Control 57:12, 1701-1708 (1996).]

13. L. Cesari. Asymptotic behavior and stability problems in ordinary differential equations. Springer, Berlin (1959).

14. I.G. Malkin. The methods of Lyapunov and Poincare in the theory of nonlinear oscillations. Editorial URSS, Moscow (2010). (in Russian).

15. A.P. Markeev. Libration points in celestial mechanics and astrodynamics. Nauka, Moscow (1978). (in Russian).

16. M. Roseau. Vibrations non linéaires et théorie de la stabilité. Springer, Berlin (1966). (in French).

17. T. Kato. Perturbation theory of linear operators. Grundlehren der mathematischen Wissenschaften. 132. Springer, Berlin (1976).

18. M.A. Krasnosel'skij, M.G. Yumagulov. The parameter functionalization method in the eigenvalue problem // Dokl. Akad. Nauk. 365:2, 162-164 (1999). [Dokl. Math. 59:2, 204-206 (1999).]

19. Yu.A. Kuznetsov. Elements of applied bifurcation theory. Springer, New York (2004).

Liliya Sunagatovna Ibragimova,

Bashkir State Agrarian University,

50-letiya Oktyabrya str. 34,

450001, Ufa, Russia

E-mail: lilibr@mail.ru

Il'mira Zhavatovna Mustafina,

Bashkir State University,

Zaki Validi str. 32,

450074, Ufa, Russia

E-mail: fanina84@bk.ru

Marat Gayazovich Yumagulov,

Bashkir State University,

Zaki Validi str. 32,

450074, Ufa, Russia

E-mail: yum_mg@mail.ru 